\title{
Existence and Uniqueness of Positive Solution for a Boundary Value Problem of Fractional Order
}

\author{
J. Caballero, J. Harjani, and K. Sadarangani \\ Departamento de Matemáticas, Universidad de Las Palmas de Gran Canaria, Campus de Tafira Baja, \\ 35017 Las Palmas de Gran Canaria, Spain \\ Correspondence should be addressed to K. Sadarangani, ksadaran@dma.ulpgc.es \\ Received 10 February 2011; Revised 9 April 2011; Accepted 16 May 2011 \\ Academic Editor: Elena Litsyn
}

Copyright (C 2011 J. Caballero et al. This is an open access article distributed under the Creative Commons Attribution License, which permits unrestricted use, distribution, and reproduction in any medium, provided the original work is properly cited.

\begin{abstract}
We are concerned with the existence and uniqueness of positive solutions for the following nonlinear fractional boundary value problem: $D_{0+}^{\alpha} u(t)+f(t, u(t))=0,0 \leq t \leq 1,3<\alpha \leq 4, u(0)=$ $u^{\prime}(0)=u^{\prime \prime}(0)=u^{\prime \prime}(1)=0$, where $D_{0+}^{\alpha}$ denotes the standard Riemann-Liouville fractional derivative. Our analysis relies on a fixed point theorem in partially ordered sets. Some examples are also given to illustrate the results.
\end{abstract}

\section{Introduction}

Differential equations of fractional order occur more frequently in different research and engineering areas such as physics, chemistry, economics, and control of dynamical. Indeed, we can find numerous applications in viscoelasticity, electrochemistry control, porous media, and electromagnetism. (see, e.g., [1-7]).

For an extensive collection of results about this type of equations we refer the reader to the monograph by Kilbas and Trujillo [8], Samko et al. [9], Miller and Ross [10], and Podlubny [11].

On the other hand, some basic theory for the initial value problems of fractional differential equations involving the Riemann-Liouville differential operator has been discussed by Lakshmikantham and Vatsala [12], Lakshmikantham [13], El-Sayed and ElMaghrabi [14], Bai [15], Bai and Ge [16], Bai and Lü [17], Zhang [18], and Kempfle et al. $[19,20]$. 
In [17] the authors studied the following two-point boundary value problem of fractional order:

$$
\begin{gathered}
D_{0^{+}}^{\alpha} u(t)+a(t) f(t, u(t))=0, \quad 0<t<1,1<\alpha \leq 2, \\
u(0)=u(1)=0,
\end{gathered}
$$

and they proved the existence of positive solutions by means of the Krasnosel'skii fixed point theorem and Leggett-Williams fixed point theorem.

In [18] the author investigated the existence of solutions of

$$
\begin{gathered}
{ }^{c} D_{0^{+}}^{\alpha} u(t)=f(t, u(t)), \quad 0<t<1,1<\alpha \leq 2, \\
u(0)=v \neq 0, \quad u(1)=\rho \neq 0 .
\end{gathered}
$$

Since boundary values are nonzero, the Riemann-Liouville fractional derivative $D_{0^{+}}^{\alpha}$ is not suitable and the author used the Caputo fractional derivative ${ }^{c} D_{0^{+}}^{\alpha}$.

Motivated by these works, in this paper we discuss the existence and uniqueness of positive solutions for the following nonlinear boundary value problem of fractional order:

$$
\begin{gathered}
D_{0^{+}}^{\alpha} u(t)+f(t, u(t))=0, \quad 0<t<1,3<\alpha \leq 4, \\
u(0)=u^{\prime}(0)=u^{\prime \prime}(0)=u^{\prime \prime}(1)=0 .
\end{gathered}
$$

This problem was studied in [21], where the authors use lower and upper solution method and the Schauder fixed point theorem which cannot ensure the uniqueness of the solution. The practical relevance of $3<\alpha \leq 4$ appears in problems related with other areas as physics and economics which can be modeled by these fractional boundary values problems. Particularly, these problems appear in the Hamiltonian formulation for the lagrangians depending on fractional derivatives of coordinates when the systems are nonconservative (see, e.g., [7]).

Our main interest in this paper is to give an alternative answer to the main results of the paper [21].

The main tool used in our study is a fixed point theorem in partially ordered sets which gives us uniqueness of the solution.

\section{Preliminaries and Previous Results}

For the convenience of the reader, we present here some definitions, lemmas and basic results that will be used in the proofs of our theorems.

Definition 2.1. The Riemann-Liouville fractional integral of order $\alpha>0$ of a function $f$ : $(0, \infty) \rightarrow \mathbb{R}$ is given by

$$
I_{0^{+}}^{\alpha} f(t)=\frac{1}{\Gamma(\alpha)} \int_{0}^{t}(t-s)^{\alpha-1} f(s) d s
$$

provided that the right-hand side is pointwise defined on $(0, \infty)$ and where $\Gamma(\alpha)$ denotes the gamma function. 
Definition 2.2. The Riemann-Liouville fractional derivative of order $\alpha>0$ of a function $f$ : $(0, \infty) \rightarrow \mathbb{R}$ is given by

$$
D_{0^{+}}^{\alpha} f(t)=\frac{1}{\Gamma(n-\alpha)}\left(\frac{d}{d t}\right)^{n} \int_{0}^{t} \frac{f(s)}{(t-s)^{\alpha-n+1}} d s,
$$

where $n=[\alpha]+1$ and $[\alpha]$ denotes the integer part of $\alpha$.

The following two lemmas can be found in $[17,22]$.

Lemma 2.3. Let $\alpha>0$ and $u \in C(0,1) \cap L^{1}(0,1)$. Then fractional differential equation

$$
D_{0^{+}}^{\alpha} u(t)=0
$$

has

$$
u(t)=c_{1} t^{\alpha-1}+c_{2} t^{\alpha-2}+\cdots+c_{n} t^{\alpha-n}
$$

for some $c_{i} \in \mathbb{R}(i=1,2, \ldots n)$ and $n=[\alpha]+1$ as unique solution.

Lemma 2.4. Assume that $u \in C(0,1) \cap L^{1}(0,1)$ with a fractional derivative of order $\alpha>0$ that belongs to $C(0,1) \cap L^{1}(0,1)$. Then

$$
I_{0^{+}}^{\alpha} D_{0^{+}}^{\alpha} u(t)=u(t)+c_{1} t^{\alpha-1}+c_{2} t^{\alpha-2}+\cdots+c_{n} t^{\alpha-n},
$$

for some $c_{i} \in \mathbb{R}(i=1, \ldots, n)$ and $n=[\alpha]+1$.

Using Lemma 2.4, in [21] the following result is proved.

Lemma 2.5. Given $f \in C[0,1]$ and $f(t) \geq 0$, the unique nonnegative solution for

$$
\begin{gathered}
D_{0^{+}}^{\alpha} u(t)+f(t)=0, \quad 0<t<1,3<\alpha \leq 4, \\
u(0)=u^{\prime}(0)=u^{\prime \prime}(0)=u^{\prime \prime}(1)=0
\end{gathered}
$$

is

$$
u(t)=\int_{0}^{1} G(t, s) f(s) d s,
$$

where

$$
G(t, s)= \begin{cases}\frac{t^{\alpha-1}(1-s)^{\alpha-3}-(t-s)^{\alpha-1}}{\Gamma(\alpha)}, & 0 \leq s \leq t \leq 1, \\ \frac{t^{\alpha-1}(1-s)^{\alpha-3}}{\Gamma(\alpha)}, & 0 \leq t \leq s \leq 1 .\end{cases}
$$


In the sequel, we present the fixed-point theorems which we will use later. These results appear in [23].

Theorem 2.6. Let $(X, \leq)$ be a partially ordered set and suppose that there exists a metric $d$ in $X$ such that $(X, d)$ is a complete metric space. Assume that $X$ satisfies the following condition

$$
\text { if }\left\{x_{n}\right\} \text { is a nondecreasing sequence in } X \text { such that } x_{n} \rightarrow x \text {, then } x_{n} \leq x \forall n \in \mathbb{N} \text {. }
$$

Let $T: X \rightarrow X$ be a nondecreasing mapping such that

$$
d(T x, T y) \leq d(x, y)-\psi(d(x, y)), \text { for } x \geq y,
$$

where $\psi:[0, \infty) \rightarrow[0, \infty)$ is a continuous and nondecreasing function such that $\psi$ is positive in $(0, \infty), \psi(0)=0$ and $\lim _{t \rightarrow \infty} \psi(t)=\infty$. If there exists $x_{0} \in X$ with $x_{0} \leq T\left(x_{0}\right)$ then $T$ has a fixed point.

Moreover, if $(X, \leq)$ satisfies the following condition:

for $x, y \in X$ there exists $z \in X$ which is comparable to $x$ and $y$,

which appears in [24], the following result is proved [23].

Theorem 2.7. Adding condition (2.11) to the hypotheses of Theorem 2.6 one obtains the uniqueness of the fixed point.

Remark 2.8. In Theorems 2.6 and 2.7 the condition $\lim _{t \rightarrow \infty} \psi(t)=\infty$ is redundant.

In our considerations we will work in the Banach space $C[0,1]=\{x:[0,1] \rightarrow \mathbb{R}$, continuous $\}$ with the standard norm $\|x\|=\sup \{|x(t)|: t \in[0,1]\}$.

Notice that this space can be equipped with a partial order given by

$$
x, y \in C[0,1], x \leq y \Longleftrightarrow x(t) \leq y(t), \quad \text { for } t \in[0,1] .
$$

In $[24]$ it is proved that $(C[0,1], \leq)$ with the classical metric given by

$$
d(x, y)=\sup _{0 \leq t \leq 1}\{|x(t)-y(t)|\}
$$

satisfies condition (2.9) of Theorem 2.6. Moreover, for $x, y \in C[0,1]$, as the function $\max (x, y) \in C[0,1],(C[0,1], \leq)$ satisfies condition $(2.11)$.

Finally, by $\mp$ we denote the class of functions $\psi:[0, \infty) \rightarrow[0, \infty)$ continuous, nondecreasing, positive in $(0, \infty)$ and $\psi(0)=0$.

By 2 we denote the class of functions $\varphi:[0, \infty) \rightarrow[0, \infty)$ continuous, nondecreasing, satisfying that $I-\varphi \in \mathcal{F}$, where $I$ denotes the identity mapping on $[0, \infty)$. 


\section{Main Result}

The main result of the paper is the following.

Theorem 3.1. Problem (1.3) has a unique positive solution $u(t)$ if the following conditions are satisfied.

(H1) $f:[0,1] \times[0, \infty) \rightarrow[0, \infty)$ is continuous and nondecreasing with respect to the second argument.

(H2) There exists $t_{0} \in[0,1]$ such that $f\left(t_{0}, 0\right)>0$.

(H3) There exists $0<\lambda \leq(\alpha-2) \Gamma(\alpha+1) / 2$ such that, for $x, y \in[0, \infty)$ with $y \geq x$ and $t \in[0,1]$,

$$
f(t, y)-f(t, x) \leq \lambda \cdot \psi(y-x)
$$

where $\psi \in 2$.

Before the proof of Theorem 3.1, we will need some properties of Green's function appearing in Lemma 2.5 .

Lemma 3.2. $G(t, s) \geq 0$, and $G$ is a continuous function on $[0,1] \times[0,1]$.

Proof. The continuity of $G$ is easily checked. In order to prove the nonnegativness of $G(t, s)$, for $0 \leq t \leq s \leq 1$, it is obvious that

$$
G(t, s)=\frac{t^{\alpha-1}(1-s)^{\alpha-3}}{\Gamma(\alpha)} \geq 0
$$

In the case of $0 \leq s \leq t \leq 1$ with $t \neq 0$, we have

$$
\begin{aligned}
G(t, s) & =\frac{1}{\Gamma(\alpha)}\left(t^{\alpha-1}(1-s)^{\alpha-3}-(t-s)^{\alpha-1}\right) \\
& =\frac{1}{\Gamma(\alpha)}\left(t^{\alpha-1}\left[(1-s)^{\alpha-3}-\left(1-\frac{s}{t}\right)^{\alpha-1}\right]\right) .
\end{aligned}
$$

As $s \leq s / t$, we have $1-s \geq 1-(s / t)$ and, consequently,

$$
(1-s)^{\alpha-3} \geq\left(1-\frac{s}{t}\right)^{\alpha-3}
$$

Taking into account that the function $g(\alpha)=t^{\alpha}$ with $\alpha>0$ and $t \in(0,1)$ is decreasing we have

$$
(1-s)^{\alpha-3} \geq\left(1-\frac{s}{t}\right)^{\alpha-3} \geq\left(1-\frac{s}{t}\right)^{\alpha-1}
$$

The last inequality and (3.3) give us $G(t, s) \geq 0$ with $t \neq 0$. Finally, notice that $G(0, s)=0$, and this finishes the proof. 
Lemma 3.3. One has

$$
\sup _{t \in[0,1]} \int_{0}^{1} G(t, s) d s=\frac{2}{(\alpha-2) \Gamma(\alpha+1)} .
$$

Proof. Since

$$
\begin{aligned}
\int_{0}^{1} G(t, s) d s & =\int_{0}^{t} G(t, s) d s+\int_{t}^{1} G(t, s) d s \\
& =\frac{1}{\Gamma(\alpha)} \int_{0}^{t}\left(t^{\alpha-1}(1-s)^{\alpha-3}-(t-s)^{\alpha-1}\right) d s+\frac{1}{\Gamma(\alpha)} \int_{t}^{1} t^{\alpha-1}(1-s)^{\alpha-3} d s \\
& =\frac{1}{\Gamma(\alpha)}\left(\frac{t^{\alpha-1}}{\alpha-2}-\frac{1}{\alpha} t^{\alpha}\right)
\end{aligned}
$$

and if we put $\varphi(t)=\int_{0}^{1} G(t, s) d s=(1 / \Gamma(\alpha))\left(\left(t^{\alpha-1}\right) /(\alpha-2)-(1 / \alpha) t^{\alpha}\right)$, then, as

$$
\varphi^{\prime}(t)=\frac{1}{\Gamma(\alpha)}\left(\frac{\alpha-1}{\alpha-2} t^{\alpha-2}-t^{\alpha-1}\right)>0, \quad \text { for } t>0
$$

we deduce that $\varphi(t)=\int_{0}^{1} G(t, s) d s$ is strictly increasing and, consequently,

$$
\begin{aligned}
\sup _{t \in[0,1]} \int_{0}^{1} G(t, s) d s & =\int_{0}^{1} G(1, s) d s=\frac{1}{\Gamma(\alpha)}\left(\frac{1}{\alpha-2}-\frac{1}{\alpha}\right) \\
& =\frac{2}{\alpha(\alpha-2) \Gamma(\alpha)}=\frac{2}{(\alpha-2) \Gamma(\alpha+1)}
\end{aligned}
$$

In the sequel, we give the proof of Theorem 3.1.

Proof of Theorem 3.1. Consider the cone

$$
P=\{u \in C[0,1]: u(t) \geq 0\}
$$

Obviously, $P$ is a closed set of $C[0,1]$, and, thus, $P$ is a complete metric space with the distance given by $d(u, v)=\sup _{t \in[0,1]}\{|u(t)-v(t)|\}$. $P$ can be equipped with a partial order defined by

$$
x, y \in P, \quad x \leq y \Longleftrightarrow x(t) \leq y(t), \text { for } t \in[0,1]
$$

Using a similar argument to that in [24], it can be proved that $(P, \leq)$ satisfies condition (2.9) of Theorem 2.6. Moreover, as for $x, y \in P$ the function $\max (x, y) \in P,(P, \leq)$ satisfies condition (2.11). 
Now, we consider the operator $T$ defined on $P$ and given by

$$
(T u)(t)=\int_{0}^{1} G(t, s) f(s, u(s)) d s, \quad \text { for } u \in P .
$$

By (H1) and Lemma 3.2, $T$ applies $P$ into itself.

In the sequel we check that $T$ satisfies the assumptions of Theorem 2.6.

Firstly, we prove that $T$ is a nondecreasing operator. In fact, by (H1), for $u, v \in P$ with $u \geq v$ and $t \in[0,1]$, we have

$$
(T u)(t)=\int_{0}^{1} G(t, s) f(s, u(s)) d s \geq \int_{0}^{1} G(t, s) f(s, v(s)) d s=(T v)(t) .
$$

Now, we prove that $T$ satisfies the contractive condition appearing in Theorem 2.6.

In fact, for $u, v \in P$ and $u \geq v$, taking into account assumption (H3), we get

$$
\begin{aligned}
d(T u, T v) & =\sup _{t \in[0,1]}\{|(T u)(t)-(T v)(t)|\}=\sup _{t \in[0,1]}((T u)(t)-(T v)(t)) \\
& =\sup _{t \in[0,1]} \int_{0}^{1} G(t, s)(f(s, u(s))-f(s, v(s))) d s \\
& \leq \sup _{t \in[0,1]} \int_{0}^{1} G(t, s) \lambda \cdot \psi(u(s)-v(s)) d s .
\end{aligned}
$$

As $\psi \in 2$ and, thus, $\psi$ is nondecreasing and by Lemma 3.3, from the last inequality we obtain

$$
\begin{aligned}
d(T u, T v) & \leq \lambda \psi(d(u, v)) \cdot \sup _{t \in[0,1]} \int_{0}^{1} G(t, s) d s \\
& =\lambda \cdot \psi(d(u, v)) \cdot \frac{2}{(\alpha-2) \Gamma(\alpha+1)} .
\end{aligned}
$$

Using the fact that $\lambda \leq 2 /(\alpha-2) \Gamma(\alpha+1)$ (assumption (H3)), we have

$$
d(T u, T v) \leq \psi(d(u, v))=d(u, v)-(d(u, v)-\psi(d(u, v))) .
$$

Put $\varphi(x)=x-\psi(x)$, As $\psi \in \mathcal{2}$, this means that $\varphi \in \mathcal{F}$. The last inequality gives us

$$
d(T u, T v) \leq d(u, v)-\varphi(d(u, v)) .
$$

This proves that $T$ satisfies the contractive condition of Theorem 2.6.

Finally, as $G(t, s) \geq 0$ (Lemma 3.2) and $f \geq 0$ (assumption (H1)), we have

$$
(T 0)(t)=\int_{0}^{1} G(t, s) f(s, 0) d s \geq 0,
$$

where 0 denotes the zero function. 
Now, Theorem 2.6 shows that problem (1.3) has at least one nonnegative solution. As $(P, \leq)$ satisfies condition (2.11), we obtain the uniqueness of the solution.

In what follows, we will prove that this solution is positive (this means that $x(t)>0$, for $t \in(0,1))$.

Finally, we will prove that the zero function is not the solution for problem (1.3). In fact, in contrary case, the zero function is a fixed point of $T$ and, thus, we have

$$
0=\int_{0}^{1} G(0, s) f(s, 0) d s, \quad \text { for } t \in[0,1]
$$

The nonnegative character of the functions $G$ and $f$ and the last expression give us

$$
G(t, s) \cdot f(s, 0)=0 \text { a.e. }(s), \text { for } t \in[0,1] \text {. }
$$

This and the fact that $G(t, s) \neq 0$ a.e. (s) for any $t \in[0,1]$ because $G(t, s)$ is given by a polynomial implies

$$
f(s, 0)=0 \quad \text { a.e. }(s) \text {. }
$$

Taking into account assumption (H2), $f\left(t_{0}, 0\right)>0$ for certain $t_{0} \in[0,1]$. By the continuity of $f$ we can find a set $A \subset[0,1]$ with $t_{0} \in A$ and $\mu(A)>0$, where $\mu$ is the Lebesgue measure, such that $f(t, 0)>0$ for $t \in A$. This contradicts (3.21).

This proves that the zero function is not the solution for problem (1.3). Now, we will prove that the solution $x$ is positive.

In the contrary case, we find $0<t^{*}<1$ such that $x\left(t^{*}\right)=0$. As the solution $x$ is a fixed point of the operator $T$, this means that

$$
x\left(t^{*}\right)=\int_{0}^{1} G\left(t^{*}, s\right) f(s, x(s)) d s=0
$$

Since $x \in P$ and, thus, $x \geq 0$ and by the fact that $f$ is nondecreasing in the second variable and $G(t, s) \geq 0$, we can get

$$
0=x\left(t^{*}\right)=\int_{0}^{1} G\left(t^{*}, s\right) f(s, x(s)) d s \geq \int_{0}^{1} G\left(t^{*}, s\right) f(s, 0) d s \geq 0
$$

and this inequality implies

$$
x\left(t^{*}\right)=\int_{0}^{1} G\left(t^{*}, s\right) f(s, 0) d s=0
$$

Using a similar reasoning to the one above used we obtain a contradiction.

Therefore, $x(t)>0$, for $t \in[0,1]$.

This finishes the proof. 
Remark 3.4. In Theorem 3.1, condition (H2) seems to be a strong condition in order to obtain a positive solution for problem (1.3), but when there is uniqueness of solution one will see that this condition is a very adjusted one. More precisely, under the assumption that problem (1.3) has a unique nonnegative solution $x(t)$ one has

$$
f\left(t_{0}, 0\right)>0 \text { for certain } t_{0} \in[0,1] \text { if and only if } x(t) \text { is a positive solution. }
$$

In fact, if $f\left(t_{0}, 0\right)>0$ for certain $t_{0} \in[0,1]$ the argument used in the proof of Theorem 3.1 give us that $x(t)$ is a positive solution.

For the other implication, suppose that $f(t, 0)=0$ for any $t \in[0,1]$. Under this assumption, our problem (1.3) admits as solutions the function $x(t)$ and the zero function and this contradicts the hypothesis about uniqueness of solution to problem (1.3). Therefore, $f\left(t_{0}, 0\right)>$ 0 for certain $t_{0} \in[0,1]$.

Remark 3.5. Notice that the assumptions in Theorem 3.1 are invariant by additive perturbations. More precisely, if $f(t, 0)=0$ for any $t \in[0,1]$ and $f$ satisfies (H1) and (H3) of Theorem 3.1, then $g(t, u)=a(t)+f(t, u)$, with $a:[0,1] \rightarrow[0, \infty)$ a nondecreasing continuous function with $a\left(t_{0}\right) \neq 0$ for certain $t_{0} \in[0,1]$, satisfies (H1), (H2), and (H3) of Theorem 3.1 and the following nonlinear boundary value problem of fractional order:

$$
\begin{gathered}
D_{0^{+}}^{\alpha} u(t)+g(t, u(t))=0, \quad 0<t<1,3<\alpha \leq 4, \\
u(0)=u^{\prime}(0)=u^{\prime \prime}(0)=u^{\prime \prime}(1)=0
\end{gathered}
$$

has a unique positive solution (by Theorem 3.1).

In the sequel we present an example where the results can be applied.

Example 3.6. Consider the fractional boundary value problem

$$
\begin{gathered}
D_{0^{+}}^{7 / 2} u(t)+\left(t^{2}+1\right) \ln (2+u(t))=0, \quad 0<t<1, \\
u(0)=u^{\prime}(0)=u^{\prime \prime}(0)=u^{\prime \prime}(1)=0 .
\end{gathered}
$$

In this case, $f(t, u)=\left(t^{2}+1\right) \ln (2+u)$ for $(t, u) \in[0,1] \times[0, \infty)$. Obviously, $f$ is a continuous function and $f(t, 0)=\left(t^{2}+1\right) \ln 2 \neq 0$ for $t \in[0,1]$. As $\partial f / \partial u=\left(t^{2}+1\right)(1 /(2+u))>0$ for $u \in[0, \infty), f$ is nondecreasing with respect to the second variable.

Besides, for $u \geq v$ and $t \in[0,1]$, we have

$$
\begin{aligned}
\left(t^{2}+1\right)[\ln (2+u)-\ln (2+v)] & =\left(t^{2}+1\right) \cdot \ln \left(\frac{2+u}{2+v}\right) \\
& =\left(t^{2}+1\right) \ln \left(\frac{2+v+u-v}{2+v}\right)=\left(t^{2}+1\right) \ln \left(1+\frac{u-v}{2+v}\right) \\
& \leq\left(t^{2}+1\right) \ln (1+(u-v)) \leq 2 \ln (1+(u-v)) .
\end{aligned}
$$

A straightforward calculation gives us that $\psi(x)=\ln (1+x)$ satisfies that $\psi \in 2$. 
Moreover, in this case $\lambda=2, \alpha=7 / 2$ and we have

$$
\frac{(\alpha-2) \Gamma(\alpha+1)}{2}=\frac{(7 / 2-2) \Gamma(7 / 2+1)}{2}=\frac{3}{4} \Gamma\left(\frac{7}{2}+1\right)=\frac{3}{4} \cdot \frac{7}{2} \cdot \Gamma\left(\frac{7}{2}\right) \approx 8.7228>2=\lambda .
$$

Finally, Theorem 3.1 proves the existence and uniqueness of a positive solution for problem (3.27).

\section{A Final Remark}

In connection with problem (1.3), the main result in [21] is the following.

Theorem 4.1 (see [21, Theorem 3.1]). Problem (1.3) has a positive solution $u(t)$ if the following conditions are satisfied:

$\left(H_{f}\right) f(t, u) \in \mathcal{C}\left([0,1] \times[0, \infty), \mathbb{R}^{+}\right)$is nondecreasing relative to $u, f(t, p(t)) \neq 0$ for $t \in(0,1)$, where $p(t)=\int_{0}^{1} G(t, s) d s=(1 / \Gamma(\alpha))\left(\left(t^{\alpha-1}\right) /(\alpha-2)-(1 / \alpha) t^{\alpha}\right)$, and there exists a positive constant $\mu<1$ such that

$$
k^{\mu} f(t, u) \leq f(t, k u), \quad \forall 0 \leq k \leq 1 .
$$

In the sequel, we present an example which can be treated by Theorem 3.1 and it cannot be covered by Theorem 4.1.

Example 4.2. Consider the fractional boundary value problem

$$
\begin{gathered}
D_{O^{+}}^{7 / 2} u(t)+\left(t^{2}+1\right)(\rho u(t)+c)=0, \quad 0 \leq t \leq 1, \\
u(0)=u^{\prime}(0)=u^{\prime \prime}(0)=u^{\prime \prime}(1)=0,
\end{gathered}
$$

with $c>0$ and $0<\rho<1$.

In this case, $f(t, u)=\left(t^{2}+1\right)(\rho u+c)$, for $(t, u) \in[0,1] \times[0, \infty)$. Obviously, $f$ is continuous and nondecreasing with respect to the second variable since $\partial f / \partial u=\rho\left(t^{2}+1\right)>0$.

Besides, if $u \geq v$ and $t \in[0,1]$, we have

$$
\begin{aligned}
f(t, u)-f(t, v) & =\left(t^{2}+1\right)[\rho u+c-(\rho v+c)] \\
& =\left(t^{2}+1\right) \rho(u-v) \leq 2 \rho(u-v) .
\end{aligned}
$$

In this case, $\psi(x)=\rho x$ and it is easily seen that $\varphi(x)=x-\psi(x)=(1-\rho) x$ belongs to $\mathcal{F}$. 
Moreover, in this case, $\lambda=2$ and, as $\alpha=7 / 2$, we have

$$
\begin{aligned}
\frac{(\alpha-2) \Gamma(\alpha+1)}{2} & =\frac{(7 / 2-2) \Gamma(7 / 2+1)}{2}=\frac{3}{4} \Gamma\left(\frac{7}{2}+1\right) \\
& =\frac{3}{4} \cdot \frac{7}{2} \cdot \Gamma\left(\frac{7}{2}\right) \approx 8.7228>2=\lambda .
\end{aligned}
$$

As $f(t, 0)=c\left(t^{2}+1\right)>0$ for any $t \in[0,1]$, Theorem 3.1 gives us the existence and uniqueness of positive solution for problem (4.2).

On the other hand, we will show that $f(t, u)=\left(t^{2}+1\right)(\rho u+c)$ with $0<\rho<1$ and $c>0$ does not satisfy $\left(H_{f}\right)$ of Theorem 4.1 . In fact, suppose that there exists $0<\mu<1$ such that

$$
k^{\mu} f(t, u) \leq f(t, k u), \quad \text { for any } 0 \leq k \leq 1
$$

This implies that

$$
k^{\mu} \leq \frac{f(t, k u)}{f(t, u)}=\frac{\left(t^{2}+1\right)(\rho k u+c)}{\left(t^{2}+1\right)(\rho u+c)}=\frac{\rho k u+c}{\rho u+c} .
$$

Notice that $\lim _{u \rightarrow \infty}(\rho k u+c / \rho u+c)=k$, and, consequently, taking limit as $u \rightarrow \infty$ in the last inequality, we get

$$
k^{\mu} \leq k,
$$

this is false because $0<\mu<1$ and the function $h(\alpha)=k^{\alpha}$ is decreasing when $0<k<$ 1. Therefore, problem (4.2) can be covered by Theorem 3.1 and it cannot be studied by Theorem 4.1.

\section{Conclusions}

Our main contribution in this paper is to prove under certain assumptions the existence and the uniqueness of positive solution for problem (1.3) which was treated in [21]. In [21] the question of uniqueness of solution was not considered. Moreover, we present an example which can be covered by the results of this paper and cannot be treated by the ones obtained in [21].

\section{Acknowledgment}

This paper was partially supported by Ministerio de Educación y Ciencia Project MTM $2007 / 65706$.

\section{References}

[1] K. Diethelm and A. D. Freed, "On the solutions of nonlinear fractional order differential equations used in the modelling of viscoplasticity," in Scientifice Computing in Chemical Engineering II: 
Computational Fluid Dynamics, Reaction Engineering and Molecular Properties, F. Keil, W. Mackens, H. Voss, and J. Werthers, Eds., pp. 217-224, Springer, Heidelberg, Germany, 1999.

[2] L. Gaul, P. Klein, and S. Kemple, “Damping description involving fractional operators," Mechanical Systems and Signal Processing, vol. 5, no. 2, pp. 81-88, 1991.

[3] W. G. Glockle and T. F. Nonnenmacher, "A fractional calculus approach to self-similar protein dynamics," Biophysical Journal, vol. 68, no. 1, pp. 46-53, 1995.

[4] F. Mainardi, "Fractional calculus: some basic problems in continuum and statistical mechanics," in Fractals and Fractional Calculus in Continuum Mechanics (Udine, 1996), C. A. Carpinteri and F. Mainardi, Eds., vol. 378 of CISM Courses and Lectures, pp. 291-348, Springer, Vienna, Austria, 1997.

[5] R. Metzler, W. Schick, H.-G. Kilian, and T. F. Nonnenmacher, "Relaxation in filled polymers: a fractional calculus approach," The Journal of Chemical Physics, vol. 103, no. 16, pp. 7180-7186, 1995.

[6] K. B. Oldham and J. Spanier, The Fractional Calculus, vol. 11 of Mathematics in Science and Engineering, Academic Press, New York, NY, USA, 1974.

[7] E. M. Rabei, K. I. Nawafleh, R. S. Hijjawi, S. I. Muslih, and D. Baleanu, "The Hamilton formalism with fractional derivatives," Journal of Mathematical Analysis and Applications, vol. 327, no. 2, pp. 891-897, 2007.

[8] A. A. Kilbas and J. J. Trujillo, "Differential equations of fractional order: methods, results and problems. I," Applicable Analysis, vol. 78, no. 1-2, pp. 153-192, 2001.

[9] S. G. Samko, A. A. Kilbas, and O. I. Marichev, Fractional Integrals and Derivatives. Theory and Applications, Gordon and Breach Science, Yverdon, Switzerland, 1993.

[10] K. S. Miller and B. Ross, An Introduction to the Fractional Calculus and Fractional Differential Equations, A Wiley-Interscience Publication, John Wiley \& Sons, New York, NY, USA, 1993.

[11] I. Podlubny, Fractional Differential Equations, vol. 198 of Mathematics in Science and Engineering, Academic Press, San Diego, Calif, USA, 1999.

[12] V. Lakshmikantham and A. S. Vatsala, "Basic theory of fractional differential equations," Nonlinear Analysis: Theory, Methods E Applications, vol. 69, no. 8, pp. 2677-2682, 2008.

[13] V. Lakshmikantham, "Theory of fractional functional differential equations," Nonlinear Analysis: Theory, Methods E Applications, vol. 69, no. 10, pp. 3337-3343, 2008.

[14] A. M. A. El-Sayed and E. M. El-Maghrabi, "Stability of a monotonic solution of a non-autonomous multidimensional delay differential equation of arbitrary (fractional) order," Electronic Journal of Qualitative Theory of Differential Equations, vol. 2008, no. 16, pp. 1-9, 2008.

[15] C. Bai, "Positive solutions for nonlinear fractional differential equations with coefficient that changes sign," Nonlinear Analysis: Theory, Methods \& Applications, vol. 64, no. 4, pp. 677-685, 2006.

[16] Z. Bai and W. Ge, "Existence of three positive solutions for some second-order boundary value problems," Computers \& Mathematics with Applications, vol. 48, no. 5-6, pp. 699-707, 2004.

[17] Z. Bai and H. Lü, "Positive solutions for boundary value problem of nonlinear fractional differential equation," Journal of Mathematical Analysis and Applications, vol. 311, no. 2, pp. 495-505, 2005.

[18] S. Zhang, "Existence of solution for a boundary value problem of fractional order," Acta Mathematica Scientia. Series B, vol. 26, no. 2, pp. 220-228, 2006.

[19] S. Kempfle, I. Schäfer, and H. Beyer, "Functional calculus and a link to fractional calculus," Fractional Calculus \& Applied Analysis, vol. 5, no. 4, pp. 411-426, 2002.

[20] S. Kempfle, I. Schäfer, and H. Beyer, "Fractional calculus via functional calculus: theory and applications," Nonlinear Dynamics, vol. 29, no. 1-4, pp. 99-127, 2002.

[21] S. Liang and J. Zhang, "Positive solutions for boundary value problems of nonlinear fractional differential equation," Nonlinear Analysis: Theory, Methods E Applications, vol. 71, no. 11, pp. 55455550, 2009.

[22] A. A. Kilbas, H. M. Srivastava, and J. J. Trujillo, Theory and Applications of Fractional Differential Equations, vol. 204 of North-Holland Mathematics Studies, Elsevier Science B.V., Amsterdam, The Netherlands, 2006.

[23] J. Harjani and K. Sadarangani, "Fixed point theorems for weakly contractive mappings in partially ordered sets," Nonlinear Analysis: Theory, Methods \& Applications, vol. 71, no. 7-8, pp. 3403-3410, 2009.

[24] J. J. Nieto and R. Rodríguez-López, "Contractive mapping theorems in partially ordered sets and applications to ordinary differential equations," Order, vol. 22, no. 3, pp. 223-239, 2005. 


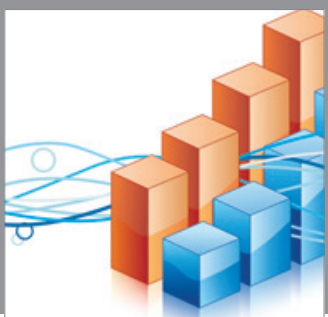

Advances in

Operations Research

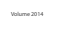

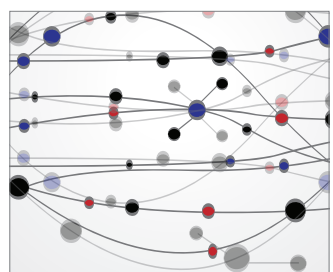

\section{The Scientific} World Journal
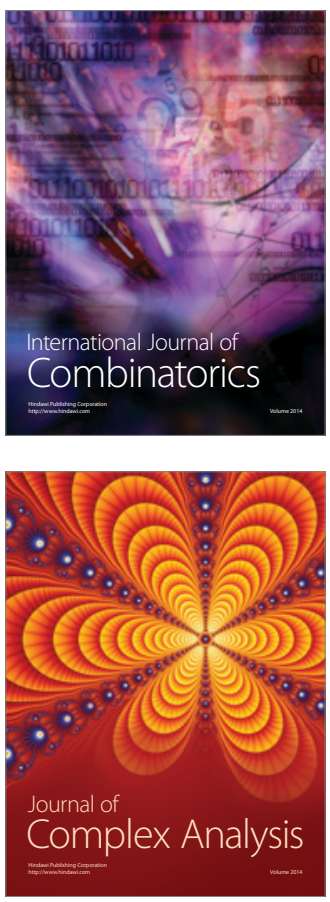

International Journal of

Mathematics and

Mathematical

Sciences
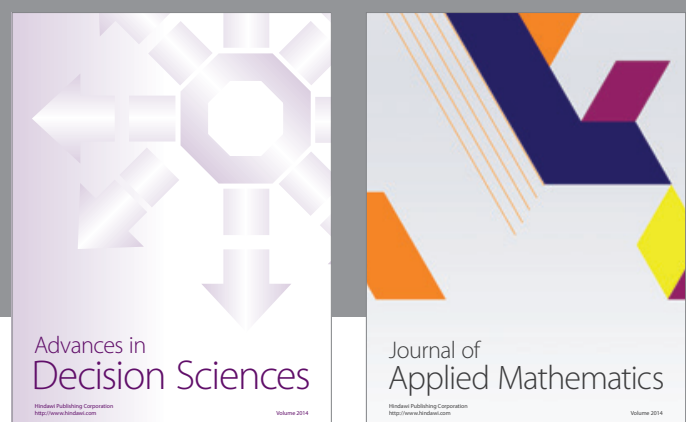

Journal of

Applied Mathematics
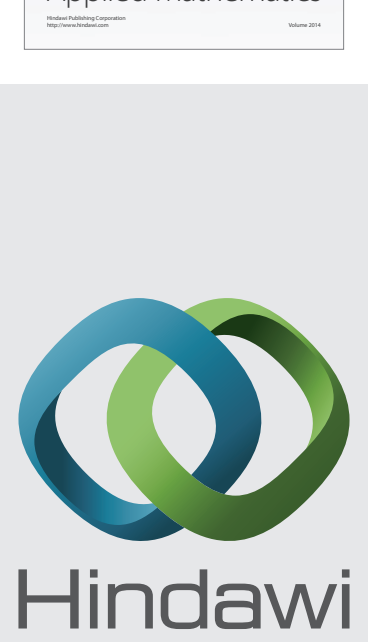

Submit your manuscripts at http://www.hindawi.com
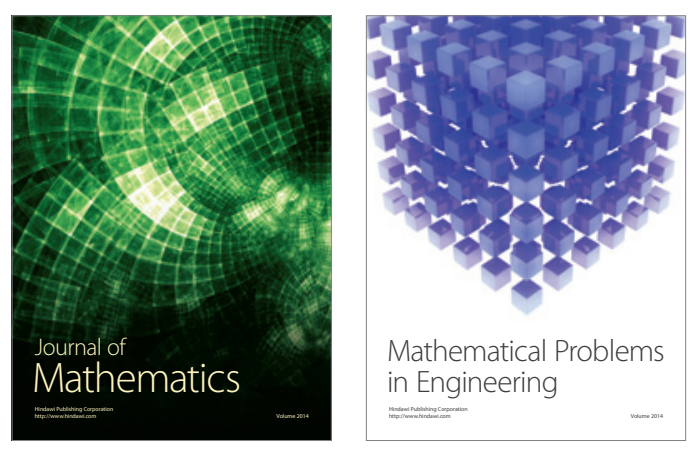

Mathematical Problems in Engineering
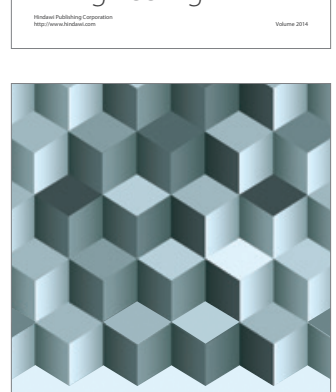

Journal of

Function Spaces
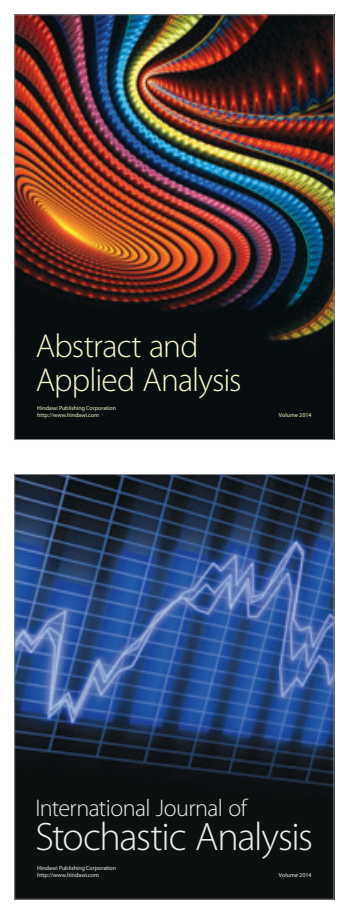

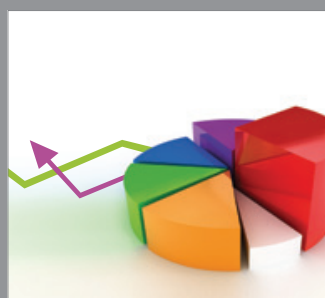

ournal of

Probability and Statistics

Promensencen
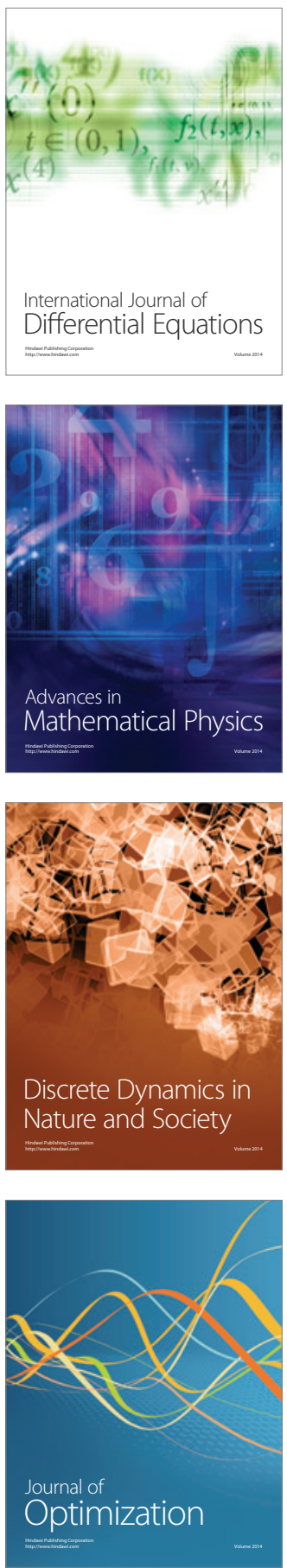